\title{
Cambios de Hábitos Alimentarios debido al confinamiento de Covid-19 en Comunidad Campesina de Sapallanga 2021
}

\author{
Changes in Eating Habits Due to the confinement of Covid-19 in the Peasant Community of Sapallanga \\ 2021
}

\author{
Belinda Olga García Inga 1,a, Marivel Rosa Martínez Véliz 1,b, Rosario Eleana Cuadros Ríos ${ }^{1, c}$, Jenny \\ Giovanna Poma Salinas 1,d, Carmela Haydee Velásquez Ledesma 1,e
}

\section{RESUMEN}

Objetivo: determinar los cambios alimentarios debido al confinamiento de la COVID-19 en la comunidad campesina de Sapallanga Huancayo 2021. Material y métodos: fue un estudio de tipo básico, con diseño no experimental de tipo descriptivo simple de corte transversal, la población estuvo conformada por 2045 personas adultas y la muestra estuvo conformada por 324 personas, para ello se hizo el muestreo de tipo probabilístico aleatorio simple, la técnica utilizada fue la encuesta y el instrumento fue el cuestionario. Resultados: se identificó que las características sociodemográficas de la población de la comunidad campesina de Sapallanga Huancayo 2021. Ha predominado la edad 30 a 40 años con 120(37\%), en el sexo el masculino con 178(54,9\%), en el grado de instrucción fue secundaria con 121(37,3\%) y en la ocupación como obrero con 228(70,4\%). Además, se identificó Se identifico que los cambios de alimentos energéticos debido al confinamiento de la COVID-19 en la población de la comunidad campesina de Sapallanga Huancayo 2021. Ha aumentado en 180(55,5\%), en consumo de alimentos constructores ha disminuido en 160(49,4\%) y el consumo de alimentos protectores Se ha mantenido en 154(47,5\%). Conclusiones: Se ha determinado que los cambios de los hábitos alimentarios debido al confinamiento de la COVID-19 en la población de la comunidad de Sapallanga Huancayo 2021, ha aumentado el consumo de alimentos energéticos con el 55,5\%, ha disminuidos el consumo de alimentos constructores con el $49,4 \%$ y se ha mantenido el consumo de alimentos protectores con el $47,5 \%$.

PALABRAS CLAVE: Hábitos alimentarios, confinamiento, COVID_19.

\section{SUMMARY}

Objective: to determine the dietary changes due to the confinement of COVID-19 in the rural community of Sapallanga Huancayo 2021. Material and methods: it was a basic study, with a non-experimental design of a simple descriptive type of cross-section, the population consisted of by 2,045 adults and the sample was made up of 324 people, for this, simple random probability sampling was done, the technique used was the survey and the instrument was the questionnaire. Results: it was identified that the sociodemographic characteristics of the population of the

\footnotetext{
Universidad Privada de Huancayo Franklin Roosevelt. Huancayo, Perú.

Docente; Lic. Enfermería; Magister; ORCID ID: 0000-0003-1500-5253 b

Docente; Lic. Enfermería; Magister; ORCID ID: 0000-0003-1992-1865

Docente; Lic. Enfermería; Magister; ORCID ID: 0000-0003-1996-1621

Docente; Lic. Enfermería; ORCID ID: 0000-0002-3325-1258

Docente; Lic. Enfermería; Magister; ${ }^{\mathrm{d}}$ Doctor; ORCID ID: 0000-0002-3720-2285
} 
rural community of Sapallanga Huancayo 2021. Age 30 to 40 years with 120 (37\%) predominated, in males with $178(54.9 \%)$, in the level of education was secondary with $121(37.3 \%)$ and in the occupation as a worker with $228(70.4 \%)$. In addition, it was identified that the changes in energy foods due to the confinement of COVID-19 in the population of the peasant community of Sapallanga Huancayo 2021. It has increased by $180(55.5 \%)$, in consumption of construction foods has decreased in 160 (49.4\%) and the consumption of protective foods has remained in 154 (47.5\%). Conclusions: It has been determined that changes in eating habits due to the confinement of COVID-19 in the population of the community of Sapallanga Huancayo 2021, has increased the consumption of energy foods with $55.5 \%$, has decreased the consumption of Builder foods with $49.4 \%$ and the consumption of protective foods with $47.5 \%$ has been maintained.

KEYWORDS: Eating habits, confinement, COVID_19.

\section{INTRODUCCIÓN}

La Organización Mundial de la Salud (OMS) la ha declarado pandemia. La humanidad está consternada, múltiples gobiernos han obligado al aislamiento total, con éxito variable debido a la negligencia de parte de la comunidad. En muchas ciudades las instituciones y el personal sanitario no son suficientes para atender la catástrofe. El aislamiento es la única estrategia eficaz para detener el crecimiento logarítmico de COVID-19. El motivo científico del aislamiento es que más del 60 $\%$ de los contagios surgen de personas asintomáticas. La enfermedad no solo produce síntomas respiratorios. El SARS-Cov-2, además, puede producir náuseas, dolor abdominal, vómito, diarrea, anosmia y ageusia. El $50 \%$ de los infectados pueden tener síntomas digestivos, que incluso preceden a los respiratorios. La ruta fecal-oral trasmite el virus, aún sin diarrea (1). El distanciamiento social incluye acciones de control de infecciones destinadas a retrasar la propagación de la enfermedad al minimizar el contacto cercano entre las personas. Los métodos incluyen cuarentenas, restricciones de viaje y cierre de escuelas, lugares de trabajo, estadios, teatros o centros comerciales. Las personas también pueden aplicar métodos de distanciamiento social limitando los viajes, evitando áreas llenas de gente y alejándose físicamente de las personas enfermas. Muchos gobiernos ahora exigen o recomiendan el distanciamiento social en las regiones afectadas por el brote (2).

Los hábitos alimentarios sanos comienzan en los primeros años de vida; la lactancia materna favorece el crecimiento sano y mejora el desarrollo cognitivo; además, puede proporcionar beneficios a largo plazo, entre ellos la reducción del riesgo de sobrepeso y obesidad y de enfermedades no transmisibles en etapas posteriores de la vida. La ingesta calórica debe estar equilibrada con el gasto calórico (3).
Hábitos alimentarios saludables para combatir el COVID-19, según la OMS. La Organización Mundial de la Salud (OMS) recomienda seguir estos 5 hábitos alimentarios a la hora de combatir el coronavirus. 1. Consume alimentos integrales y mantén una dieta diversa. Esta es la mejor manera de asegurarse de consumir suficientes vitaminas y minerales para mantener un sistema inmunológico saludable. 2. Siempre incluye frutas y verduras en tu plato. 3. Come nueces como bocadillo. Los frutos secos son ricos en vitamina $\mathrm{E}$, un antioxidante conocido por su capacidad para estimular el sistema inmunológico. 4. Agrega granos integrales y legumbres. 5. Incorpora alimentos de origen animal estos pueden proporcionar una variedad de micronutrientes que son difíciles de obtener en cantidades adecuadas solo a partir de alimentos de origen vegetales (4).

Relacionado al estudio se han encontrado algunos antecedentes nacionales que se menciona a continuación Guillen en su trabajo titulado Percepción y hábitos de alimentación durante la cuarentena por COVID-19 en el Perú; 2020. Resultados: el 84,4 \% de los encuestados tenían entre 18 a 35 años y el 53,1 $\%$ eran del sexo femenino. Educación superior técnica era el grado de instrucción predominante. Los cambios más frecuentes se refieren a mayor consumo de frutas y verduras ( $32 \%$ ), y reducción en el consumo productos lácteos como yogurt, leche y queso (22\%). Productos alimenticios de mediana prioridad, como bebidas industrializadas y productos de confitería, mostraron una reducción de $68 \%$. Posterior a la compra de alimentos, el $45 \%$ de encuestados, higienizan los productos con desinfectantes de uso doméstico. Para el $55 \%$ de encuestados, una alimentación saludable fortalece el sistema inmunológico y reduce la propensión a la infección por coronavirus (5).

Así mismo se han identificado algunos antecedentes internacionales que describimos a continuación: 
Diaz A, Barker S. realizaron un estudio titulado: Impacto del cambio en los hábitos alimenticios sobre la salud de la población entre los 18 y 30 años por el confinamiento obligatorio en Colombia; 2021. Resultados: en Colombia, la población a pesar de verse afectada económicamente, presentó un alto consumo de alimentos. Conclusiones: la población colombiana durante la cuarentena por la crisis del COVID-19, presentó dificultades económicas considerables que contribuyeron a una pérdida de rutina respecto al cambio de hábitos alimenticios (6).

Tormo et al., realizaron un estudio titulado: Cambios en las practicas culinarias y gastronómicas durante el confinamiento de la COVID-19 en la provincia de Alicante- España, 2020 (7). En el cual el $70 \%$ cocinó con más frecuencia y dedicó más tiempo a hacerlo. Más del $35 \%$ amplió sus conocimientos culinarios con un incremento de consulta a la web y redes sociales. Durante el confinamiento predominó la cocina tradicional valenciana y los postres fueron las elaboraciones preferidas. El cocinado en grupo aumentó en más de 11 puntos porcentuales, al igual que comer en compañía, aunque persiste el uso de dispositivos audiovisuales durante el acto de comer. El $84 \%$ modificó su percepción sobre algún aspecto relacionado con la alimentación. Conclusiones: durante el confinamiento, el aumento de la actividad culinaria se tradujo en una apuesta por la cocina tradicional en detrimento de los platos precocinados, más convivialidad en el acto de comer y un mayor compromiso con el comercio y el consumo de alimentos de proximidad y la sustentabilidad del sistema alimentario (7).

Álvarez Frealizo un estudio titulado: Hábitos alimentarios y actividad física durante el confinamiento por COVID-19 en estudiantes de la facultad de cienciaspontificia Universidad Javeriana Bogotá Colombia; 2020. Los resultados no demostraron cambios en el consumo de algunos de grupos de alimentos, sin embargo, se evidenció un aumento en el consumo de comidas rápidas $(32,1 \%)$, frutas $(43 \%)$ y verduras $(40,9 \%)$. En cambio, el $37,9 \%$ de los estudiantes aumentaron su peso. Como conclusión, los estudiantes implementaron ciertos hábitos alimentarios saludables como preparar sus comidas, aumentar el consumo de frutas y la actividad física. Sin embargo, aspectos negativos como el aumento de peso también fueron observados (8).

Ramírez A., Zerpa C. realizaron un estudio titulado: relaciones entre confinamiento domiciliario por la pandemia COVID-19: hábitos y estados emocional en personas con o sin conductas sugestivas de trastornos del comportamiento alimentario residentes de zonas urbanas. Venezuela; 2020. Los resultados sugieren anticipar las consecuencias para la salud mental y física que tienen las políticas públicas de confinamiento domiciliario en la población general y, en particular, prever las necesidades de atención y orientación que las personas con TCA demandarán durante el periodo que dure una cuarentena (9).

Federick et al., realizaron un estudio titulado: Hábitos alimentarios y COVID. Análisis descriptivo durante el aislamiento social en Argentina; 2020. Las prácticas alimentarias se describieron según la frecuencia de consumo semanal de los principales grupos de alimentos. Resultados: del total de encuestados el $73,9 \%$ fueron mujeres y el $26,0 \%$ hombres, un $72,2 \%$ de los encuestados alcanzó niveles de estudios universitarios completo o incompleto. Se destacó una baja frecuencia de consumo diario de: leche $44,5 \%$; carnes $11,5 \%$, frutas $44,5 \%$ y verduras $50,5 \%$. Conclusión: Es necesario el diseño e implementación de intervenciones efectivas que promuevan activamente el consumo de leche y derivados, carnes, vegetales y frutas, aun para poblaciones con alto niveles de escolaridad (10).

Pérez et al., realizaron un estudio titulado: cambios en los hábitos alimentarios durante el periodo de confinamiento por la pandemia COVID-19 en España; 2020. Resultados: los cambios más frecuentes se refieren a mayor consumo de fruta $(27 \%)$, huevos $(25,4 \%)$, legumbres $(22,5 \%)$, verduras $(21 \%)$ y pescado $(20 \%)$ y reducción en el consumo de carnes procesadas $(35,5 \%)$, cordero o conejo $(32 \%)$, pizza (32,6\%), bebidas alcohólicas destiladas (44,2\%), bebidas azucaradas $(32,8 \%)$ o chocolate $(25,8 \%)$, el $24,6 \%$ pasa sentado más de 9 horas diarias y el $30,7 \%$ de las personas fumadoras (14,7\%) fuma más. Un $37 \%$ refiere no dormir bien. Conclusiones: Los participantes en este estudio refieren cambios alimentarios en el periodo de confinamiento en España con tendencia hacia mayor consumo de alimentos saludables, menor consumo de alimentos de menos interés nutricional y aumento de la práctica de cocinar en casa (11).

Severi y Medina realizaron un estudio titulado cambios en los hábitos alimentarios y actividad física durante el aislamiento social durante el COVID-19: estudio descriptivo sobre una muestra de trabajadores. Uruguay; 2020. Resultados: la tercera parte de los trabajadores $(30,6 \%)$ perciben incremento de 
peso, y cambiaron su comportamiento, casi el $47 \%$ manifiesta que come más o con mayor frecuencia, respecto al tipo de alimentos la mayor parte refiere que incrementaron alimentos ricos en carbohidratos y ultra procesados. Conclusión: existen cambios de comportamiento alimentario y de actividad física no saludables y que promueven el riego de enfermedades crónicas no transmisibles. Se sugiere tomar medidas que acompañen las ya realizadas para contener la transmisión del coronavirus en la comunidad (12).

Miranda et al., encontratron que de una muestra compuesta por un $30 \%$ de hombres y un $70 \%$ de mujeres, tendencia hacia el consumo de alimentos saludables, como frutas y verduras, y un descenso de otros menos saludables, como son los alimentos procesados. Un $46 \%$ de la población indicó estar comiendo más que antes del confinamiento, aumentando el picoteo entre horas ( $54 \%$ ) y la ansiedad por la comida, debido a situaciones de estrés o nervios, en un $40 \%$ de los encuestados. Conclusiones: durante el confinamiento, los hábitos alimentarios y el estilo de vida de la población gallega se han visto modificados. El incremento de peso experimentado en una población que ya partía de un elevado porcentaje de sobrepeso y obesidad al inicio del confinamiento podría tener un impacto negativo en el desarrollo de patologías crónicas no transmisibles (13).

Hábitos alimentarios: Un hábito es un proceso gradual y se adquiere a lo largo de los años mediante una práctica repetida, un hábito alimentario son patrones de consumo de alimentos que se han enseñado o se van adoptando paulatinamente de acuerdo a los gustos y preferencias. Los hábitos alimentarios saludables, son aquellas prácticas de consumo de alimentos por medio de las cuales las personas seleccionan su alimentación en función de mantener una buena salud. Esto incluye adoptar un patrón de consumo que incluya todos los grupos de alimentos, según lo enseñan las guías alimentarias y además respetar horarios y tiempos de comida. Por lo tanto, el escenario escolar se convierte en un espacio que permite la promoción de hábitos alimentarios saludables, no solo dentro de los salones de clases, sino también apoyando la teoría con las diversas actividades que se viven en los centros educativos. Una adecuada alimentación, en calidad y cantidad, es indispensable para el crecimiento ya que aportará los nutrientes que se relacionan con la formación de los tejidos y la energía necesaria para el buen funcionamiento de los órganos, además funcionará como factor de prevención de las enfermedades que pueden ser causadas por la deficiencia o exceso de alimentos (14).

Hábitos alimentarios saludables: El desayuno es la comida más importante del día, debe ser completo y equilibrado y aportar fruta, lácteos, pan o cereales. Dedícale al menos 10 o 15 minutos. Una dieta equilibrada, debe ser lo más variada posible, es decir, no elimines ningún grupo completo de alimentos, hay que comer de todo. Lo menos conveniente lo reducirás a días especiales, pero comer saludable no significa no poder probar jamás una onza de chocolate. Date un pequeño capricho una vez a la semana, por ejemplo. Los hidratos de carbono, sobre todo los de lenta asimilación, como son los que nos aportan los cereales, pasta, arroz, o pan, (mejor integrales) y las patatas y las legumbres, deben ser una parte importante de la dieta, al menos la mitad de lo que comes (15).

Alimentos energéticos: cuando los alimentos son ricos en hidratos de carbono y/o grasas, se les clasifica como alimentos energéticos. Estas sustancias al ser metabolizadas, proporcionan la energía que el organismo necesita para mantener las funciones y realizar las actividades habituales. Las proteínas también pueden ser utilizadas en caso de emergencia, pero la obtención de energía no resulta tan beneficiosa desde el punto de vista metabólico (16). Función energética: físicamente, para efectuar cualquier tipo de transformación o movimiento se insume energía. Por ello nuestro organismo requiere energía y una reserva de la misma. Las necesidades de energía, provienen de la oxidación en las células de los principios inmediatos: hidratos, grasas, y también del alcohol. Esa energía es utilizada fundamentalmente para la síntesis proteica, y para el transporte activo de sustancias a través de la membrana celular. Si bien suponíamos que las proteínas aportaban energía por clasificarse como orgánicas, la energía que aportan es una cantidad mínima, por lo tanto, no las identificamos como fuente principal de energía (16).

Alimentos reguladores: contienen vitaminas y minerales, además de fibra 3 a $6 \mathrm{~g} /$ día y agua $2 \mathrm{~L} /$ día, y permiten a nuestro cuerpo utilizar adecuadamente las sustancias nutritivas de los alimentos energéticos y constructores. Es necesario consumirlos en abundancia dos veces al día para prevenir diversas enfermedades y evitar estreñimiento, gases y flatulencias. Existen evidencias de que un alto consumo de verduras y frutas reduce el riesgo de contraer cáncer de mama, estómago e intestino (17). Función reguladora: se vincula con la utilización adecuada de las sustancias 
estructurales y energéticas; esta es la función principal de las vitaminas y las sales minerales (18).

Alimentos constructores: son ricos en proteínas favorecen la formación de nuevos tejidos, la conservación de la piel y la renovación constante de células internas. Mantienen un adecuado nivel de defensa contra las enfermedades infecciosas. Lo dice su nombre son constructores y por lo tanto ayudan al crecimiento de niños y adolescentes talla y desarrollo de órganos en general y a la formación de masa muscular, tan deseada por los deportistas. Los alimentos plásticos son aquellos ricos en proteínas y minerales (18). Las proteínas son una fuente importante de energía para tus músculos. Deben estar en una proporción del 10$15 \%$ del total de calorías. Las proteínas pueden ser de origen animal, (carne, pescados, huevos) o de origen vegetal como los cereales o las legumbres. Lleva un equilibrio entre los dos tipos. Las grasas, mejor que sean de origen vegetal, como el aceite de oliva, o de girasol. En total no deberán superar el $30 \%$ de las calorías diarias totales (15). Su función principal de las proteínas consiste en formar los tejidos del organismo durante el crecimiento y también reponer el continuo desgaste de los tejidos ya existentes. Los minerales Como el calcio participan sobre todo en la formación de huesos (18).

El objetivo del presente trabajo fue determinar los cambios alimentarios debido al confinamiento de la COVID-19 en la comunidad campesina de Sapallanga Huancayo 2021.

\section{MATERIAL Y MÉTODOS}

El tipo de investigación que se realizo fue básico con diseño de investigación no experimental de tipo descriptivo simple y de corte transversal. El estudio se realizó en la población de Sapallanga de la provincia de Huancayo de la Región Junín, para la selección de los sujetos de investigación se ha tenido el padrón de las personas del Barrio San Antonio que hacia un

Tabla 1. Cambios de los hábitos alimentarios debido al confinamiento de la COVID-19 en la población de la comunidad campesina de Sapallanga Huancayo 2021.

\begin{tabular}{lccc}
\hline Categorías & Muestra & Frecuencia & Porcentaje \\
\hline $\begin{array}{l}\text { Ha aumentado el consumo de alimentos energéticos (harinas, } \\
\text { cereales, tubérculos, arroz, grasas) }\end{array}$ & 324 & 180 & 55,5 \\
$\begin{array}{l}\text { Ha disminuido el consumo de alimentos protectores (carnes, } \\
\text { leche, menestras) }\end{array}$ & 324 & 160 & 49,4 \\
$\begin{array}{l}\text { Se ha mantenido el consumo de alimentos protectores } \\
\text { (frutas, verduras, líquidos) }\end{array}$ & 324 & 154 & 47,5 \\
\hline
\end{tabular}

Fuente: cuestionario de recolección de datos

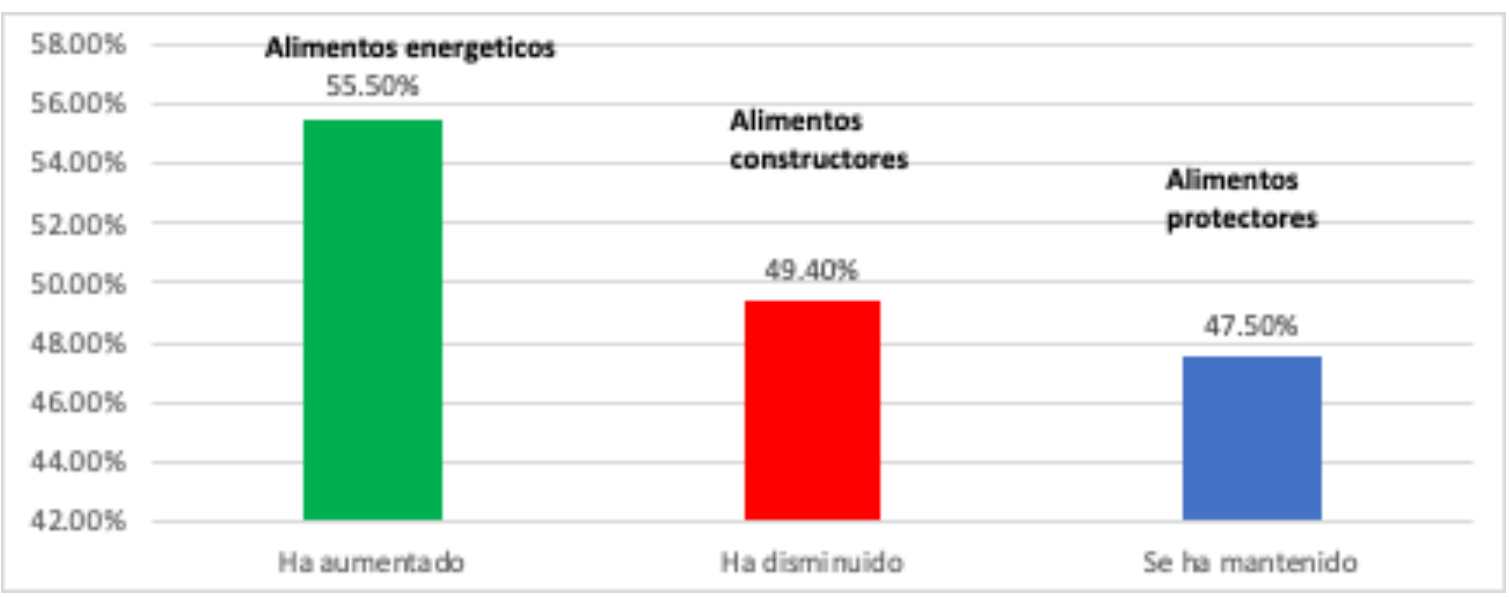

Gráfico 1. Cambios de los hábitos alimentarios debido al confinamiento de la COVID-19 en la población de la comunidad campesina de Sapallanga Huancayo 2021 
total de 2045 personas y se ha realizado un sorteo para seleccionar a los sujetos. Las pruebas estadísticas que se han utilizado para el análisis de los resultados ha sido taba de frecuencias con el apoyo del SPSSv25.

\section{RESULTADOS}

En la tabla 1 y grafico 1 se observa de $324(100 \%)$ de adultos encuestados sobre los cambios de los hábitos alimentarios debido al confinamiento de la COVID-19 en la población de la comunidad campesina de Sapallanga que $180(55,5 \%)$ refieren que ha aumentado el consumo de alimentos energéticos como son harinas, cereales, tubérculos, arroz y grasas, $160(49,5 \%)$ refieren que ha disminuido el consumo de alimentos protectores como carnes, leche, menestras y $154(47,5 \%)$ refieren que se ha mantenido el consumo de alimentos protectores como frutas, verduras, líquidos durante el confinamiento.

\section{DISCUSIÓN}

Los resultados del objetivo general representada en la tabla 1 y grafico 1 se observa de $324(100 \%)$ de adultos encuestados sobre los cambios de los hábitos alimentarios debido al confinamiento de la COVID-19 en la población de la comunidad campesina de Sapallanga que $180(55,5 \%)$ refieren que ha aumentado el consumo de alimentos energéticos como son harinas, cereales, tubérculos, arroz y grasas, $160(49,5 \%)$ refieren que ha disminuido el consumo de alimentos protectores como carnes, leche, menestras y $154(47,5 \%)$ refieren que se ha mantenido el consumo de alimentos protectores como frutas, verduras, líquidos durante el confinamiento. Estos resultados son similares al estudio realizado por Álvarez F. Quien concluyo que se evidenció un aumento en el consumo de comidas rápidas $(32,1 \%)$, también son similares al estudio realizado por Miranda et al, quienes concluyen que durante el confinamiento, los hábitos alimentarios y el estilo de vida de la población gallega han visto modificados. Sin embargo, son diferentes al estudio realizado por Pérez et al., quienes concluyen que los participantes en este estudio refieren cambios alimentarios en el periodo de confinamiento en España con tendencia hacia mayor consumo de alimentos saludables, menor consumo de alimentos de menos interés nutricional y aumento de la práctica de cocinar en casa.

Según el Ministerio de Educación Pública de Costa Rica define a los hábitos alimentarios como un proceso gradual y se adquiere a lo largo de los años mediante una práctica repetida, un hábito alimentario son patrones de consumo de alimentos que se han enseñado o se van adoptando paulatinamente de acuerdo a los gustos y preferencias. Los hábitos alimentarios saludables, son aquellas prácticas de consumo de alimentos por medio de las cuales las personas seleccionan su alimentación en función de mantener una buena salud. Esto incluye adoptar un patrón de consumo que incluya todos los grupos de alimentos, según lo enseñan las guías alimentarias y además respetar horarios y tiempos de comida.

En los resultados del objetivo específico 1, el $324(100 \%)$ adultos encuestados de la población de la comunidad campesina de Sapallanga que la edad que predomina se encuentra entre 30 a 40 años con $120(37 \%)$, y el sexo predominante es el masculino con $178(54,9 \%)$, con relación el grado de instrucción el más frecuente es secundaria con $121(37,3 \%)$ y la ocupación más frecuente es obrero con $228(70,4 \%)$. Estos resultados son similares al estudio realizado por Miranda et al., quienes concluyen que la muestra compuesta por un $30 \%$ de hombres y un $70 \%$ de mujeres (13). Por otro lado, estos resultados son diferentes al estudio realizado por Guillen quien concluye que el $84,4 \%$ de los encuestados tenían entre 18 a 35 años y el 53,1 \% eran del sexo femenino con grado de instrucción superior técnica era el más predominante (5). También son diferentes al estudio realizado por Federick et al., quienes concluyen que del total de encuestados el 73,9\% fueron mujeres y el $26,0 \%$ hombres (10).

Según la OMS, los factores sociodemográficos son todas las características asignadas a la edad, sexo, educación, ingresos, estado civil, trabajo, religión, tasa de natalidad, tasa de mortalidad, tamaño de la familia. Esto se hace para cada miembro de la población.

Los resultados de objetivo específico 2 el $324(100 \%)$ adultos encuestados en la población de la comunidad de Sapallanga que $180(55,5 \%)$ refieren que ha aumentado el consumo de alimentos energéticos debido al confinamiento, $90(27,8 \%)$ refieren que ha disminuido el consumo de alimentos energéticos debido al confinamiento $44(13,6 \%)$ refieren que se ha mantenido el consumo de alimentos energéticos debido al confinamiento de la COVID-19 y 10(3,1\%) refieren que no han consumido. Estos resultados son similares al estudio realizado por Severi y Medina, quienes concluyen que la tercera parte de los trabajadores $(30,6 \%)$ perciben incremento de peso, y cambiaron su comportamiento, casi el $47 \%$ manifiesta que come más o con mayor frecuencia alimentos ricos 
en carbohidratos y ultra procesados. Por otro lado, estos resultados son diferentes al estudio realizado por Pérez et al., quienes concluyen que hubo reducción en el consumo de bebidas azucaradas $(32,8 \%)$ o chocolate $(25,8 \%)(11)$.

Según García los alimentos energéticos cuando son ricos en hidratos de carbono y/o grasas, se les clasifica como alimentos energéticos (16), estas sustancias al ser metabolizadas, proporcionan la energía que el organismo necesita para mantener las funciones y realizar las actividades habituales. Las proteínas también pueden ser utilizadas en caso de emergencia, pero la obtención de energía no resulta tan beneficiosa desde el punto de vista metabólico. Así mismo refiere que la función energética: físicamente, para efectuar cualquier tipo de transformación o movimiento se insume energía. Por ello nuestro organismo requiere energía y una reserva de la misma. Las necesidades de energía, provienen de la oxidación en las células de los principios inmediatos: hidratos, grasas, y también del alcohol. Esa energía es utilizada fundamentalmente para la síntesis proteica, y para el transporte activo de sustancias a través de la membrana celular.

En los resultados del objetivo específico 3, el $324(100 \%)$ adultos encuestados en la población de la comunidad de Sapallanga que 160(49,4\%) refieren que ha disminuido el consumo de alimentos constructores debido al confinamiento de la COVID-19, 111(34,3\%) refieren que se ha mantenido el consumo de alimentos constructores debido al confinamiento, 38(11,7\%) refieren que ha aumentado el consumo de alimentos constructores debido al confinamiento y 15(4,6\%) refieren que no han consumido. Estos resultados son similares al estudio realizado por Guillen, quien concluye que hubo reducción del consumo de productos lácteos como yogurt, leche y queso en un (22\%). Estos resultados son similares al estudio realizado por Federick et al., quienes concluyen que se destacó una baja frecuencia de consumo diario de: leche 44,5\%; carnes 11,5\% (10). También son similares al estudio realizado por Pérez C, Gianzo M, Gotzone $\mathrm{B}$ y et al. Quienes concluyen que hubo reducción en el consumo de carnes procesadas $(35,5 \%)$, cordero o conejo $(32 \%)$.

Según Guzmán et al., refieren que los alimentos constructores son ricos en proteínas favorecen la formación de nuevos tejidos, la conservación de la piel y la renovación constante de células internas (18). Mantienen un adecuado nivel de defensa contra las enfermedades infecciosas. Lo dice su nombre son constructores y por lo tanto ayudan al crecimiento de niños y adolescentes talla y desarrollo de órganos en general y a la formación de masa muscular, tan deseada por los deportistas. Los alimentos plásticos son aquellos ricos en proteínas y minerales. Su función principal de las proteínas consiste en formar los tejidos del organismo durante el crecimiento y también reponer el continuo desgaste de los tejidos ya existentes.

En los resultados del objetivo específico 4, el $324(100 \%)$ adultos encuestados en la población de la comunidad de Sapallanga que 154(47,5\%) refieren que se ha mantenido el consumo de alimentos protectores debido al confinamiento de la COVID-19, 120(37,1\%) refieren que ha disminuido el consumo de alimentos protectores debido al confinamiento, 42(12,9\%) refieren que ha aumentado el consumo de alimentos protectores debido al confinamiento y 8(2,5\%) refieren que no han consumido. Estos resultados son similares al estudio realizado por Guillen J. quien concluye que los cambios más frecuentes se refieren a mayor consumo de frutas y verduras (32\%). También es similar al estudio realizado por Álvarez F. Quien concluye que no demostraron cambios en el consumo de algunos de grupos de alimentos como frutas (43\%) y verduras $(40,9 \%)$. Sin embargo, es diferente al estudio realizado por Federick et al., quienes concluyen se destacó una baja frecuencia de consumo diario de frutas $44,5 \%$ y verduras $50,5 \%$ (10).

Según Pérez, los alimentos reguladores o protectores contienen vitaminas y minerales, además de fibra 3 a $6 \mathrm{~g} /$ día y agua $2 \mathrm{~L} /$ día, y permiten a nuestro cuerpo utilizar adecuadamente las sustancias nutritivas de los alimentos energéticos y constructores. Es necesario consumirlos en abundancia dos veces al día para prevenir diversas enfermedades y evitar estreñimiento, gases y flatulencias. Existen evidencias de que un alto consumo de verduras y frutas reduce el riesgo de contraer cáncer de mama, estómago e intestino.

\section{CONCLUSIONES}

Se ha determinado que los cambios de los hábitos alimentarios debido al confinamiento de la COVID-19 en la población de la comunidad de Sapallanga Huancayo 2021, ha aumentado el consumo de alimentos energéticos en un 55,5\%, ha disminuidos el consumo de alimentos constructores en un $49,4 \%$ y se ha mantenido el consumo de alimentos protectores con el $47,5 \%$. 
Se identificó que las características sociodemográficas de la población de la comunidad campesina de Sapallanga Huancayo 2021. Ha predominado la edad 30 a 40 años con 120(37\%), en el sexo el masculino con $178(54,9 \%)$, en el grado de instrucción fue secundaria con $121(37,3 \%)$ y en la ocupación como obrero con 228(70,4\%).

Se identificó que los cambios de alimentos energéticos debido al confinamiento de la COVID-19 en la población de la comunidad campesina de Sapallanga Huancayo 2021. Ha aumentado en $180(55,5 \%)$.

Se identificó que los cambios de alimentos constructores debido al confinamiento de la COVID-19 en la población de la comunidad campesina de Sapallanga Huancayo 2021. Ha disminuido en $160(49,4 \%)$.

Se identificó que los cambios de alimentos protectores debido al confinamiento de la COVID-19 en la población de la comunidad campesina de Sapallanga Huancayo 2021. Se ha mantenido en $154(47,5 \%)$.

\section{Correspondencia:}

Belinda Olga García Inga

Correo electrónico: bgarcia@uroosevelt.edu.pe

\section{REFERENCIAS BIBLIOGRAFICAS}

1. Otero W, Gomez M, Ruiz O, et al. Procedimientos edoscopiscos y pandemia COVOD-19. Consideraciones basicas. Revista Colombiana de Gastroenterologia. 2020; 35(1). (Citado 19 de marzo del 2021). Disponible en: https://revistagastrocol. com/index.php/rcg/article/view/526

2. Wikipedia. Covid -19. España: Wikipedia; 2021 (Citado 19 de marzo del 2021). Disponible en: https:// es.wikipedia.org/wiki/COVID-19

3. Organización Mundial de la Salud. Alimentacion sana. Ginebra: Organización Mundial de la Salud; 2020. (Citado 19 de marzo del 2021). Disponible en: https://www.who.int/es/news-room/fact-sheets/ detail/healthy-diet

4. Organización Mundial de la Salud. Habitos alimentarios saludables para combatir el COVID-19. Ginebra: Organización Mundial de la Salud; 2020 (Citado 19 de marzo del 2021). Disponible en:: https:// www.msn.com/es-xl/video/watch/h\%C3\%A1bitosalimentarios-saludables-para-combatir-el-covid-19seg\%C3\%BAn-la-oms/vp-BB1d8OMY

5. Guillen J. Percepción y hábitos de alimentación durante la cuarentena por COVID-19 en el Perú; 2020. Revista de Investigaciones de la Universidad Le Cordon Bleu; 2021; 8(2).. DOI: https://doi. org/10.36955/RIULCB.2021v8n1.009

6. Diaz A, Barker S. Impacto del cambio en los hábitos alimenticios sobre la salud de la población entre los 18 y 30 años por el confinamiento obligatorio en Colombia. Bpgpta: Universidad EAN; 2021. (Citado 19 de marzo del 2021). Disponible en: https:// repository.ean.edu.co/bitstream/handle/ 10882/10266/DiazAngely2020.pdf? sequence= $1 \&$ isAllowed $=\mathrm{y}$

7. Tormo-Santamaría M, Català-Oltra L, PeretóRovira A, Ruíz-García A, Bernabeu-Mestre J. Cambios en las prácticas culinarias y gastronómicas durante el confinamiento de la COVID-19 en la provincia de Alicante. Rev Esp Nutr Hum Diet. 2021; 25 (Supl. 2): e1130. DOI: 10.14306/renhyd.25. S2.1130

8. Álvarez F. Hábitos alimentarios y actividad física durante el confinamiento por COVID-19 en estudiantes de la Dacultad de ciencias- pPontificia Universidad Javeriana. Tesis para título de Nutricionista. Bogotá Colombia: Pontifica Universidad Javeriana; 2020. (Citado 19 de marzo del 2021). Disponible en: https://repository.javeriana. edu.co/handle/10554/51991

9. Ramírez A, Zerpa C. Relaciones entre confinamiento domiciliario por la pandemia COVID-19: hábitos y estados emocional en personas con o sin conductas sugestivas de trastornos del comportamiento alimentario residentes de zonas urbanas de Venezuela. Sapiens Reseach. 2020; 10(2). (Citado 19 de marzo del 2021). Disponible en: https://www.srg.com.co/ bcsr/index.php/bcsr/article/view/396

10. Federick M, Calderón C, Degastaldi V, et al. Hábitos alimentarios y COVID. Análisis descriptivo durante el aislamiento social en Argentina. Nutr Clín Diet Hosp. 2020; 40(3):84-9. DOI: 10.12873/403federik

11. Pérez C, Gianzo M, Gotzone B, et al Cambios en los habitos alimentarios durante el periodo de confinamiento por la pandemia COVID-19 en España. Revista Española de Nutrición Comunitaria. 2020; 26(2):101-111.DOI:10.14642/RENC.2020.26.2.5213

12. Severi C, Medina M. Cambios en los hábitos alimentarios y actividad física durante el aislamiento físico durante el COVID -19:.Anales De La Facultad De Medicina. 2020; 7(1), e2020v7n1a15. (Citado 19 de marzo del 2021). Disponible en: http:/www. anfamed.edu.uy/index.php/rev/article/view/459

13. Miranda M, Cepeda A, Cardelle A. hábitos alimentarios en la población gallega durante el confinamiento por la COVID-19. Nutrición Hospitalaria. 2021; 37(6). (Citado 19 de marzo del 2021). Disponible en: https:// scielo.isciii.es/scielo.php?script $=$ sci_arttext\&pid 
$=\mathrm{S} 0212-16112020000800015$

14. Ministerio de Educacion Publica. Habitos alimentarios saludables [internet] Costa Rica: Ministerio de Educacion Publica; 2020 (Citado 19 de marzo del 2021). Disponible en: https://www.mep. go.cr/noticias/habitos-alimentarios-saludables

15. AXA Health Keeper. Aprende a comer saludable. Blog Heal Keper; 2019. (Citado 19 de marzo del 2021). Disponible en: https://www.axahealthkeeper. com/blog/habitos-alimentarios-saludables/
16. Garcia A. Dietética y nutrición. Educaguia; 2019. (Citado 19 de marzo del 2021). Disponible en: www. educaguia.com/apuntesde

17. Pérez M. Alimento. (Citado 19 de marzo del 2021). Disponible en: https://www.wealthmaster.us/pemundo-acurio/?user $=190.236 .205 .80 \&$ camp $=840 \& d$ evice $=$ Desktop\&brand $=$ Bitcoin System\&

18. Guzmán C, Peña F, Guerrero $M$, et al. Nutrición clínica adulto USS. Universidad San Sebastián; 2018.

Recibido: 24/04/2021

Aceptado: 19/09/2021 